\title{
Atomic Simulation of the Melting and Mechanical Behaviors of Silicon Nanowires
}

\author{
Dandan Zhao ${ }^{1,2}$, Jing $\mathrm{Li}^{2,3}$ and Lin Zhang ${ }^{1,2, *}$ \\ 1 Key Laboratory for Anisotropy and Texture of Materials (Ministry of Education), Northeastern University, \\ Shenyang 110819, China; zdd_lucifer@163.com \\ 2 Department of Materials Physics and Chemistry, School of Materials Science and Engineering, Northeastern \\ University, Shenyang 110819, China; 1810147@stu.neu.edu.cn \\ 3 The State Key Laboratory of Rolling and Automation, Northeastern University, Shenyang 110819, China \\ * Correspondence: zhanglin@imp.neu.edu.cn; Tel.: +86-24-83678479; Fax: +86-24-83683674
}

Citation: Zhao, D.; Li, J.; Zhang, L. Atomic Simulation of the Melting and Mechanical Behaviors of Silicon Nanowires. Crystals 2021, 11, 1010. https://doi.org/10.3390/cryst11091010

Academic Editors: Yougen Hu and Siya Huang

Received: 13 July 2021

Accepted: 19 August 2021

Published: 24 August 2021

Publisher's Note: MDPI stays neutral with regard to jurisdictional claims in published maps and institutional affiliations.

Copyright: (c) 2021 by the authors. Licensee MDPI, Basel, Switzerland. This article is an open access article distributed under the terms and conditions of the Creative Commons Attribution (CC BY) license (https:/ / creativecommons.org/licenses/by/ $4.0 /$ )

\begin{abstract}
Molecular dynamics simulations using a three-body potential show that the melting and mechanical behaviors of silicon nanowires are strongly dependent on their cross-section area. For the wire with a small cross-section area, rearrangements of surface atoms greatly affect thermal stability in a relatively low temperature regime. For these wires with a relatively large area, while some surface atoms adjust their positions, most of the interior atoms hold their tetrahedra packing patterns. At a high temperature, the accumulation of structural disorder can quickly extend into the entire wire, which resembles the melting of the bulk phase. By applying the uniaxial tensile, these silicon nanowires present the typical mechanical behavior of plastic materials. The atomic local stress in the necking region is apparently larger than that outside of the necking region. As the cross-section area becomes large, both the yield strength and tensile strength increase. With the increasing temperature, the elasticity decreases significantly.
\end{abstract}

Keywords: silicon nanowires; mechanical behaviors; molecular dynamics; surface

\section{Introduction}

Silicon nanowires (Si NWs) present distinguished properties differing from their bulk counterparts owing to their high surface/volume ratio [1]. During the last two decades, the Si NWs have been widely considered as suitable units, which can be integrated in functional devices including biosensors [2,3], thermoelectrics [4,5], transistors [6], resonators [7] and electrode materials for energy storage devices [8]. When these nanowires are used in the nano-electromechanical system (NEMS) as active components, such as nanorelays, nanoresonators, or switches (ON-OFF devices), the mechanical properties play an essential role in the reliability and manufacturability of these nano-devices under processing and working conditions. Therefore, it has become increasingly important to understand the mechanical properties of these nanowires, and many investigations have been reported on mechanical behaviors, including the elasticity [9-11], anelasticity [12], plasticity [13,14] and strength [15] of these components.

Upon heating these nanowires, the transition information of structures or mechanical properties is provided in terms of changes in atomic packing, as well as atomic level stress. The tensile test is an effective approach to probing the mechanical properties of materials. Over the past decades, a large number of experimental studies have investigated the mechanical properties of silicon nanowires via tensile tests [16-18]. However, unlike the mechanical testing of bulk materials, the testing of NWs heavily depends on the experimental setup, and so there are significant challenges during manipulating, which makes accurately applying and measuring the external force of strains at the nano-scale extremely difficult. In addition, there are discrepancies between observations during the experimental process, especially in regard to the plastic behaviors of nanowires. Therefore, 
computational approaches can be used to calculate the mechanical properties of reasonably sized NWs [19-21]. Among these mechanical properties, Young's modulus has been measured to study the size and temperature effect on the structures of the nanowires by utilizing many experimental approaches based on the Atomic Force Microscope (AFM), Electron Microscope (EM) or Optical Microscope (OM) [22-24]. Moreover, theoretical modeling suggests that the Young's modulus of the Si NWs decreases when the diameter and temperature decreases [25-27].

Previous reports were concentrated on the Si NWs with different sizes. For instance, the molecular-doped Si NWs, which are integrated in solar cells, have diameters of up to $70 \mathrm{~nm}$ [28]. The Si NWs as a sensing nanomaterial have dimensions in the range of $\approx 1-100 \mathrm{~nm}$, hence making them very comparable to and compatible with the dimensional scale of biological and chemical species [29]. Wang et al. covered the processing of Si NWsbased field-effect transistors (FETs), ranging from 10 to $20 \mathrm{~nm}$ wide [30]. In addition, doping provides an optimal performance of NWs. Keeping the size of the nanowire unchanged, by doping the nanowire with a controllable dopant distribution, the obtained doped nanowire can be tuned to have the characteristics of electrons in the laboratory. When comparing the local stress distribution of pure nanowires, the doped nanowires have local stress changes at the doped sites, which is critical for a device design with a reproducible electronic performance at the nanoscale [31]. However, as requirements increase significantly for the miniaturization and portability of devices, it becomes essential to investigate high-quality Si NWs with smaller-diameter cross sections. Such knowledge also helps us to improve our understanding of the relationship between the structure and properties of nano-objects with high surface/volume ratios. Therefore, a number of both theoretical and experimental investigations have been stimulated naturally to predict the structures and properties of smaller silicon nanowires. Yet, many reports on small-sized nanowires tend to examine the geometric structure and electronic properties of the Si NWs through first-principle calculations [26,32], which are a prohibited computational expense. At the nanoscale, as the number of atoms in a system increases, there is an exponential increase in computational cost, as well as an increase in data complexity. Computer simulations based on empirical potentials, such as molecular dynamics (MD), are particularly well-suited to characterizing microscopic details in these systems, involving the combined behaviors of atom movements and locally structural rearrangements, as well as stress changes at the atomic scale.

The present work is focused on changes in the packing configurations and mechanical behaviors of [001]-oriented nanowires with diameters of $0.693 \mathrm{~nm}, 0.859 \mathrm{~nm}$ and $1.034 \mathrm{~nm}$, by using atomic simulations under the tension of the heating process. The effects of crosssection area and temperature on thermal stability and mechanical behaviors were mainly observed through the analysis of energy; atomic level stress; pair distribution function; and the relationship between moduli, stress and strain, as well as atomic packing images.

\section{Simulation Approach}

The interactions among the atoms are described by the Stilinger-Weber (SW) potential $[33,34]$. This potential has been widely used to predict the molecular structure and the mechanical properties of silicon nanomaterials. The total potential energy of an $\mathrm{N}$-atom system takes the following form:

$$
E_{t o t}=\sum_{i<j} \phi_{i j}\left(r_{i j}\right)+\sum_{i<j<k} \varphi_{i j k}
$$

where $\phi_{i j}$ and $\varphi_{i j k}$ represent the potential energy of two bodies and three bodies, respectively, which can be calculated by the following form:

$$
\begin{gathered}
\phi\left(r_{i j}\right)=\varepsilon f_{2}\left(\frac{r_{i j}}{\sigma}\right) \\
\varphi_{i j k}=\varepsilon f_{3}\left(\frac{r_{i}}{\sigma}, \frac{r_{j}}{\sigma}, \frac{r_{k}}{\sigma}\right)
\end{gathered}
$$


where $\varepsilon$ and $\sigma$ are the dimensional parameters of energy and length, and $f_{2}$ and $f_{3}$ are functions of two-body and three-body potentials related to distance, respectively:

$$
\begin{gathered}
f_{2}(r)=\left\{\begin{array}{c}
A\left(B r^{-p}-r^{-q}\right) \exp \left(\frac{1}{r-a}\right), \\
0<a \\
0, \quad r \geq a
\end{array}\right. \\
f_{3}=\left\{\begin{array}{cc}
\left(r_{i}, r_{j}, r_{k}\right)=h\left(r_{i j}, r_{i k}, \theta_{j i k}\right)+h\left(r_{j i}, r_{j k}, \theta_{i j k}\right)+h\left(r_{k i}, r_{k j}, \theta_{i k j}\right), & r<a \\
0, & r \geq a
\end{array}\right.
\end{gathered}
$$

and the angle-related function is:

$$
h_{3}\left(r_{i j}, r_{i k}, \theta_{j i k}\right)=\lambda \exp \left[\frac{\gamma}{r_{i j}-a}+\frac{\gamma}{r_{i k}-a}\right] \times\left(\frac{1}{3}+\cos \theta_{j i k}\right)^{2}
$$

where $\theta_{j i k}$ is the angle between the $r_{i}$ and $r_{k}$ subtended vertex $i$, etc. The values of $\sigma, \varepsilon, A$, $B, \lambda, \Gamma, p, q$ and $a$ are adjustment parameters. The value of $a$ is chosen so that the potential is truncated between the first-nearest-neighbor and the second-nearest-neighbor distances. These parameters in the empirical potential are obtained by using the experimental and quantum mechanics calculations, as listed in Table 1.

Table 1. The parameter of empirical potential.

\begin{tabular}{lccccccccc}
\hline Parameter & $\sigma(\mathbf{n m})$ & $\varepsilon(\mathrm{kcal} / \mathrm{mol})$ & $\boldsymbol{A}$ & $\boldsymbol{B}$ & $\boldsymbol{\lambda}$ & $\boldsymbol{\Gamma}$ & $\boldsymbol{p}$ & $\boldsymbol{q}$ & $\boldsymbol{a}$ \\
\hline Value & 0.20951 & 50 & 7.049556 & 0.602225 & 21.0 & 1.20 & 4 & 0 & 1.80 \\
\hline
\end{tabular}

Initially, we built three Si NWs with different diameters in circle cross-sections, which were labeled as SiNW1, SiNW2 and SiNW3, respectively. The orientation and the cross-section of these silicon nanowires are depicted in Figure 1. The figure shows projections separately along the $\mathrm{x}, \mathrm{y}$, and $\mathrm{z}$ directions, corresponding to the [100], [010] and [001] directions. The diameters of the wires are $0.693 \mathrm{~nm}, 0.859 \mathrm{~nm}$ and $1.034 \mathrm{~nm}$, which include 450, 690 and 970 atoms in the simulation cells. To neglect the interaction between periodic images, the vacuum spaces of $100 \AA$ and $100 \AA$ are used in lateral directions for these wires, respectively.

(a)

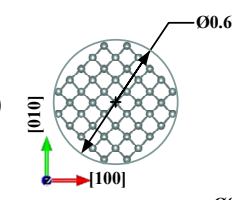

(b)

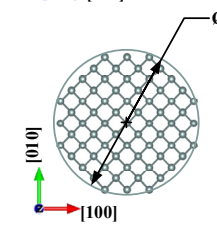

(c)

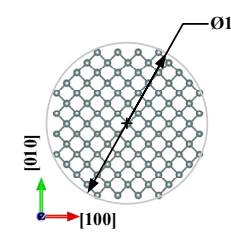

\section{mo}

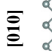
$\longrightarrow$ [0011
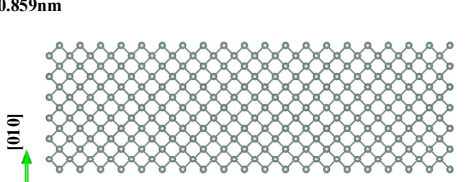

$\rightarrow$ 10011

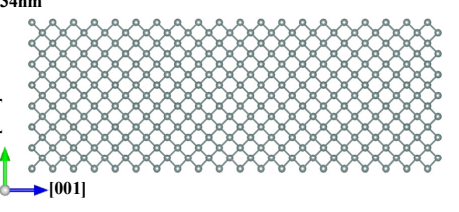

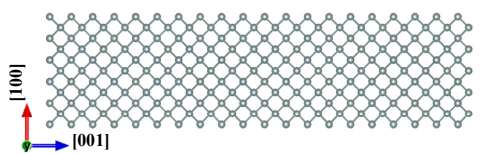
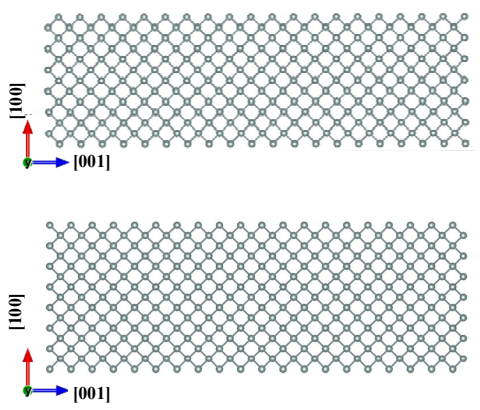

Figure 1. Initially constructed Si NWs with diameters of (a) SiNW1: $0.693 \mathrm{~nm}$, (b) SiNW2: $0.859 \mathrm{~nm}$, (c) SiNW3: $1.034 \mathrm{~nm}$. 
To observe and analyze the atomic packing structures of these Si NWs, the pair distribution function $g(r)$ provides the probability of finding the atom pairs within a distance $r$ in the system:

$$
g(r)=\frac{1}{n^{2}}\left\langle\sum_{i} \sum_{j \neq 1} \delta\left(r-r_{i j}\right)\right\rangle
$$

where $n$ is the number of atoms of the nanowires in simulation, and \langle\rangle denotes the average value for the statistical time step.

Figure 2 shows the $g(r)$ curves of these constructed initial structures. As shown in this figure, the first peak is at $0.2370 \mathrm{~nm}$, corresponding to the position of the first neighboring atom in the crystals, which is also the bond length of the crystalline silicon. The positions of the other peaks correspond to the second, third, and fourth neighbors in these nanowires, respectively. The differences in peak heights indicate that the number of paired atoms is different at this distance between a pair of atoms. The high peak indicates the large number of the paired atoms, and the low peaks represent the small numbers. The $g(r)$ curves in this figure suggest the same packing patterns of the atoms in these initial nanowires [32].

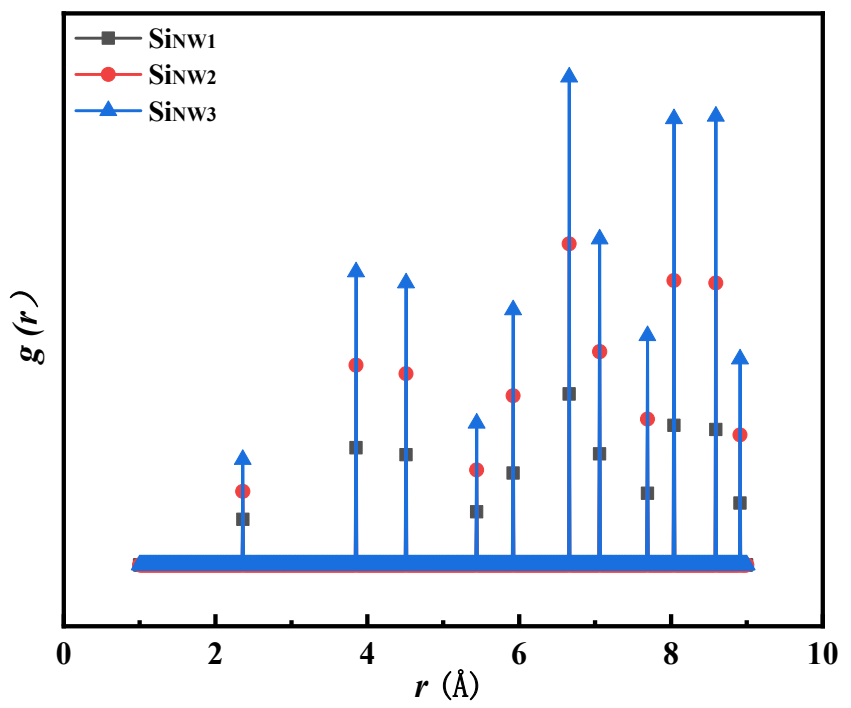

Figure 2. Pair distribution functions $g(r)$ of the three Si NWs before relaxation.

The simulations, which employed the General Utility Lattice Program (GULP) software package [35], were performed by starting with the optimal structure at $300 \mathrm{~K}$, then gradually increasing the temperature to $1600 \mathrm{~K}$ at an increment of $50 \mathrm{~K}$ in the NVT ensemble [36], where an Andersen thermostat was used to control temperature. By solving Newton's motion equations, we could obtain the positions and velocities of each atom, and a predictor-corrector algorithm was used to integrate equations of motion. At each temperature, the runs took 60,000 time steps, and the last 30,000 time steps recorded the atomic trajectories and energy, that is, they were used to calculate the statistical average values for these nanowires. Throughout simulations, a time step of $1 \mathrm{fs}$ was used.

The potential energy $E_{a v}$ per atom at each temperature is defined below:

$$
E_{a v}=\frac{1}{N}\left\langle E_{t o t}\right\rangle
$$

where $N$ is the number of atoms in the system.

As given in Equation (1), both the two-body potential energy and three-body potential energy contributes to the total potential energy of the simulated system. If the structures of the atomic packing change, the distance and angles among the atoms change accordingly, resulting in changes to the two- or three-body potential energies. Figure 3 shows a similar behavior regarding the changes of the potential energy for these nanowires at room temper- 
ature. Initially, the energy has very high values. The reason for this phenomenon is that the atoms in an original structure are packed into the same structure as in the bulk crystalline, possessing a high energy. However, because the surface atoms of nanowires are less bound by their surrounding atoms than these in the interior, they only need a small amount of energy to rearrange their packing patterns. Then, in rows of buckled dimers observed on the surfaces of Si NWs, the energy drops abruptly, and enters into the oscillating regime, implying that the nanowire is in equilibrium. Table 2 lists the surface/volume ratio for each simulated nanowire, which is calculated from the atom number in the surface divided by the total number of atoms in one nanowire. As the diameters of the cross-sections for these nanowires increase, the ratio of surface/volume decreases. Therefore, the SiNW1 with the smallest diameter has the highest value because of a high proportion of atoms in the surface, and the energy decreases as the diameter increases.

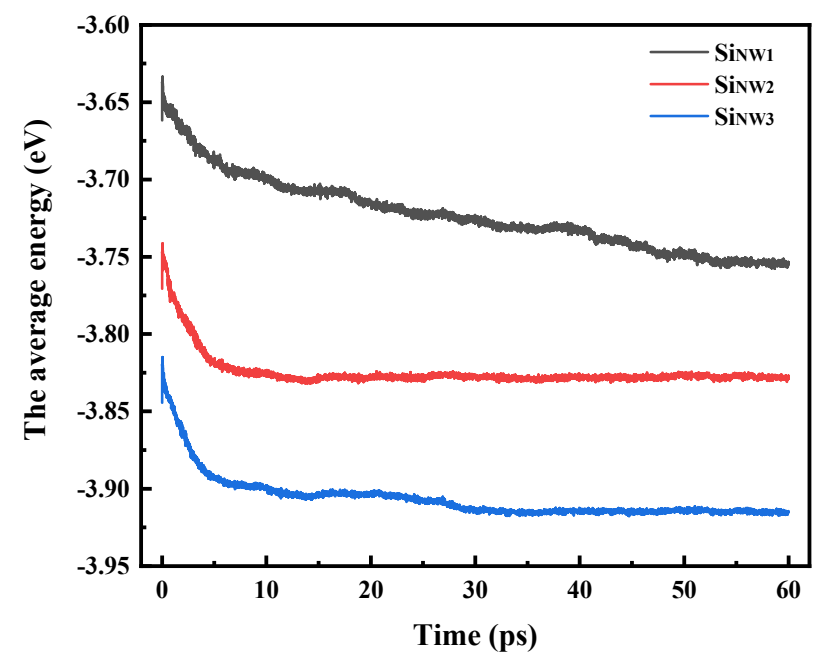

Figure 3. The variation of average potential energy of each atom for all the Si NWs with time.

Table 2. The statistics of the surface atoms number; the surface/volume ratio of all the Si NWs before relaxation at room temperature.

\begin{tabular}{ccc}
\hline & Surface Atoms Number & The Surface/Volume Ratio (S/V) \\
\hline SiNW1 & 200 & 0.444 \\
SiNW2 & 240 & 0348 \\
SiNW3 & 280 & 0.289 \\
\hline
\end{tabular}

In applying the strain for the nanowires, the cell length along the [001] axial direction of the nanowires, increases by $1 \%$ of the initial length at every $1 \mathrm{ps}$, corresponding to a strain rate of $1 \times 10^{10} \mathrm{~s}^{-1}$.

The local stress tensors $\sigma_{i}$ of each atom $i$ is given in the following equation:

$$
\sigma_{i}^{a b}=\frac{1}{V_{i}} \sum_{j \neq i} \frac{\partial E_{i}}{\partial r_{i j}} \frac{r_{i j}{ }^{a} r_{i j}{ }^{b}}{r_{i j}}
$$

where $V_{i}$ is the volume of atoms, $E_{i}$ is the energy of the $i$ th atom, and $r_{i j}{ }^{a}$ and $r_{i j}{ }^{b}$ are the Cartesian components of the vector $r_{i j}$ in which $a$ and $b$ stand for $\mathrm{x}, \mathrm{y}$, and $\mathrm{z}$. Furthermore, the isotropic atomic pressure $P_{i}$ is related to the $\sigma_{i}$ as given by the following form:

$$
P_{i}=\frac{1}{3}\left(\sigma_{i}^{x x}+\sigma_{i}^{y y}+\sigma_{i}^{z z}\right)
$$

The elastic properties of materials can be represented by Young's modulus. The modulus is derived from the slope in the elastic stage of a stress-strain curve. 


\section{Results and Discussion}

Changes of potential energy indicate changes in the packing structure in the simulated systems. A difference value of atomic potential energy is introduced in the present work, which is defined as the value obtained from the difference between the energy of two adjacent temperatures divided by the temperature difference. Figure 4 shows the variation of the difference value in the temperatures for these three nanowires upon heating. As shown in this figure, the Si NWs with smaller diameters (SiNW1) possess the most apparent oscillation changes compared with other nanowires at lower temperatures, implying apparent changes in the atomic packing of this wire. At higher temperatures, there is a sharp increase in the energy difference, specifically at temperatures of $1200 \mathrm{~K}, 1300 \mathrm{~K}$, and $1500 \mathrm{~K}$, and the abrupt change at the highest temperature for the SiNW3 indicates the need to absorb the most heat. For the SiNW1, a large proportion of the atoms occupy surface positions, which present movements with only a small amount of energy. Therefore, the position adjustments of these surface atoms significantly affect the changes in the energy differences, as shown in Figure 4. In a temperature regime from $650 \mathrm{~K}$ to $850 \mathrm{~K}$, the difference value enters into a plateau, implying that the atoms hold their packing patterns formed at $650 \mathrm{~K}$. As the temperature increases, the different values change in an oscillating mode, and then in the temperature range from $1100 \mathrm{~K}$ to $1200 \mathrm{~K}$, increases significantly, suggesting that the atomic packing becomes disordered. For the SiNW2, until $600 \mathrm{~K}$, most of the atoms hold their packing patterns. Then, the oscillating changes imply that some atoms continuously adjust their positions as the temperature increases. At high temperatures, the oscillating amplitude increases. At $1300 \mathrm{~K}$, the jump peak suggests that the atoms pack disorderly after absorbing enough heat. In the case of SiNW3, most atoms hold their packing patterns until $1200 \mathrm{~K}$. Then, a certain number of the atoms begin to change their positions, and at $1500 \mathrm{~K}$, the wire melts. It can also be seen from the Figure 4, the bulk_Si hold their packing patterns in the range from $300 \mathrm{~K}$ to $1600 \mathrm{~K}$.

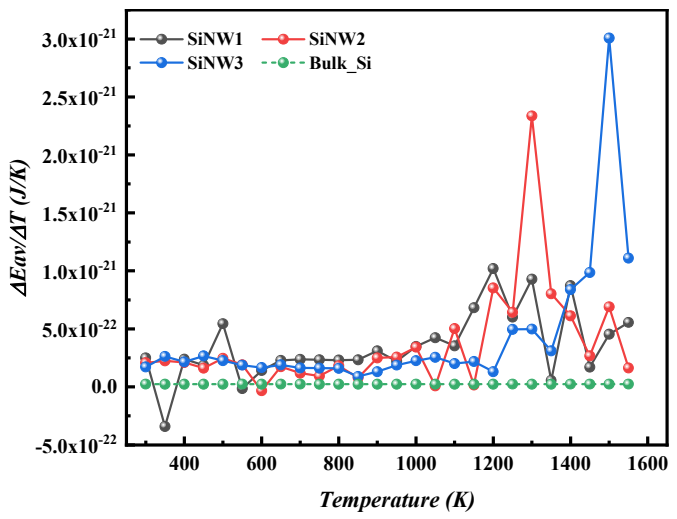

Figure 4. The difference of potential energy per atom as a function of temperatures.

As shown in Figure 5, the $g(r)$ has a characteristic of discrete peaks with a certain width, resulting from the thermal movement of atoms around their equilibrium position. It should be noted that the highest position of the first peak of the distribution function of the wires with different sizes at different temperatures, is about $0.235 \mathrm{~nm}$, corresponding to the bond length between atoms in the silicon tetrahedra. Compared with the $g(r)$ of the initial structures, peak width occurs because of the intensified thermal motion of the silicon nanowires in these atoms. When the distances between the atoms exceed the limited distance, the packing patterns change, resulting in the differences of these peak positions. Meanwhile, since the coordination number of surface atoms is lower than that of internal atoms, nearly all rearrangement occurs in the surface atoms in relatively low temperature regimes. As the temperature is in the high-temperature regimes, some distinct peaks of the nanowires disappear, indicating that the transition from the ordered structure to the disordered structure occurs. 


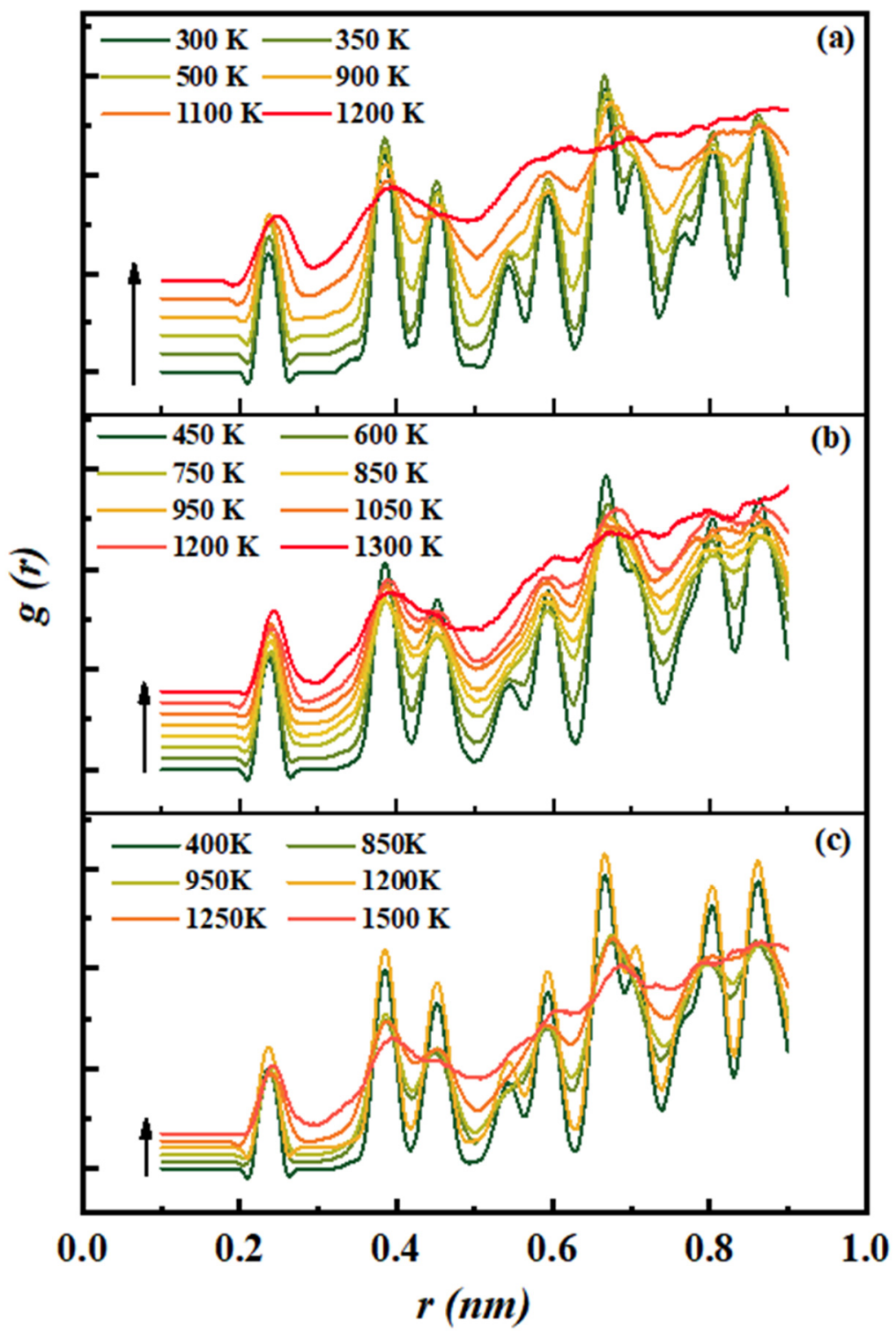

Figure 5. The $g(r)$ of the nanowires after structural relaxation at different temperatures: (a) SiNW1, (b) SiNW2, (c) SiNW3.

Figure ?? shows the packing patterns of the three nanowires at different temperatures. At relatively low temperatures, a general scene is that only a few of surface atoms adjust their positions, and most of the atoms in these three nanowires can hold their packing patterns in large temperature ranges. As the temperature increases, more surface atoms change their positions, which results in the views of these three nanowires from the axis direction getting closer to a circle, whereas most of the interior atoms are in the lattice positions. As shown by the images of the lateral section along the [001] direction, as the temperature increases, some surface atoms rearrange their packing patterns. For SiNW1, a certain number of red surface atoms are in a suspended state at $350 \mathrm{~K}$, whereas at $400 \mathrm{~K}$, these suspended atoms form dimers, resulting in the release of energy as illustrated by the apparent decrease in Figure 3. With the increase in temperature from $500 \mathrm{~K}$ to $550 \mathrm{~K}$, some dimers formed on the surface are broken, resulting in an increase in energy. Similarly, apparent surface reconstruction can be observed in SiNW2 and SiNW3 in the relatively high temperature regimes, whereas most atoms hold their packing patterns until melting at $1300 \mathrm{~K}$ and $1500 \mathrm{~K}$. 
(a) Sinwı

\begin{tabular}{|c|c|c|}
\hline Temperature & Cross section & Lateral section \\
\hline \multicolumn{3}{|l|}{$350 \mathrm{~K}$} \\
\hline \multicolumn{3}{|l|}{$400 K$} \\
\hline \multicolumn{3}{|l|}{$500 \mathrm{~K}$} \\
\hline \multicolumn{3}{|l|}{$550 \mathrm{~K}$} \\
\hline \multicolumn{3}{|l|}{$1250 \mathrm{~K}$} \\
\hline 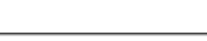 & & \\
\hline
\end{tabular}

(b) Sinw2

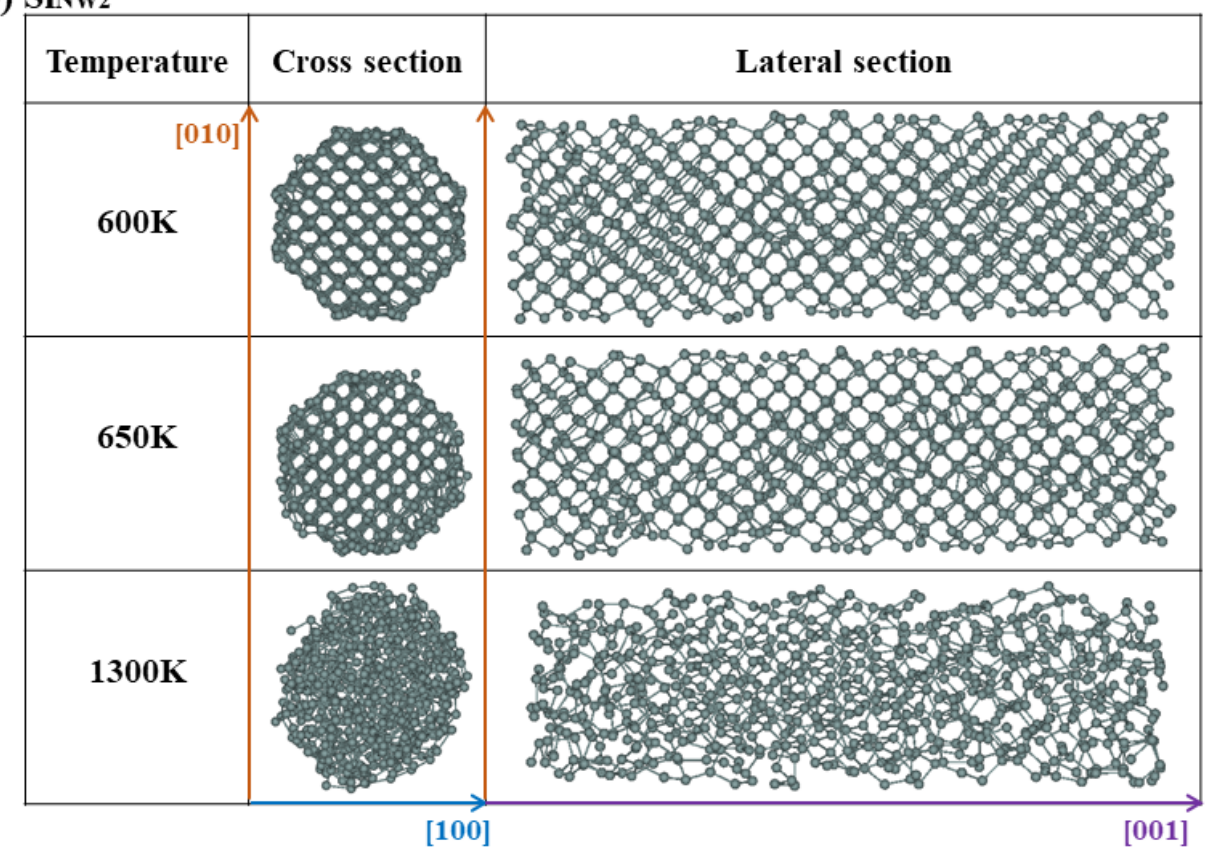

Figure 6. Cont. 
(c) Sinw3

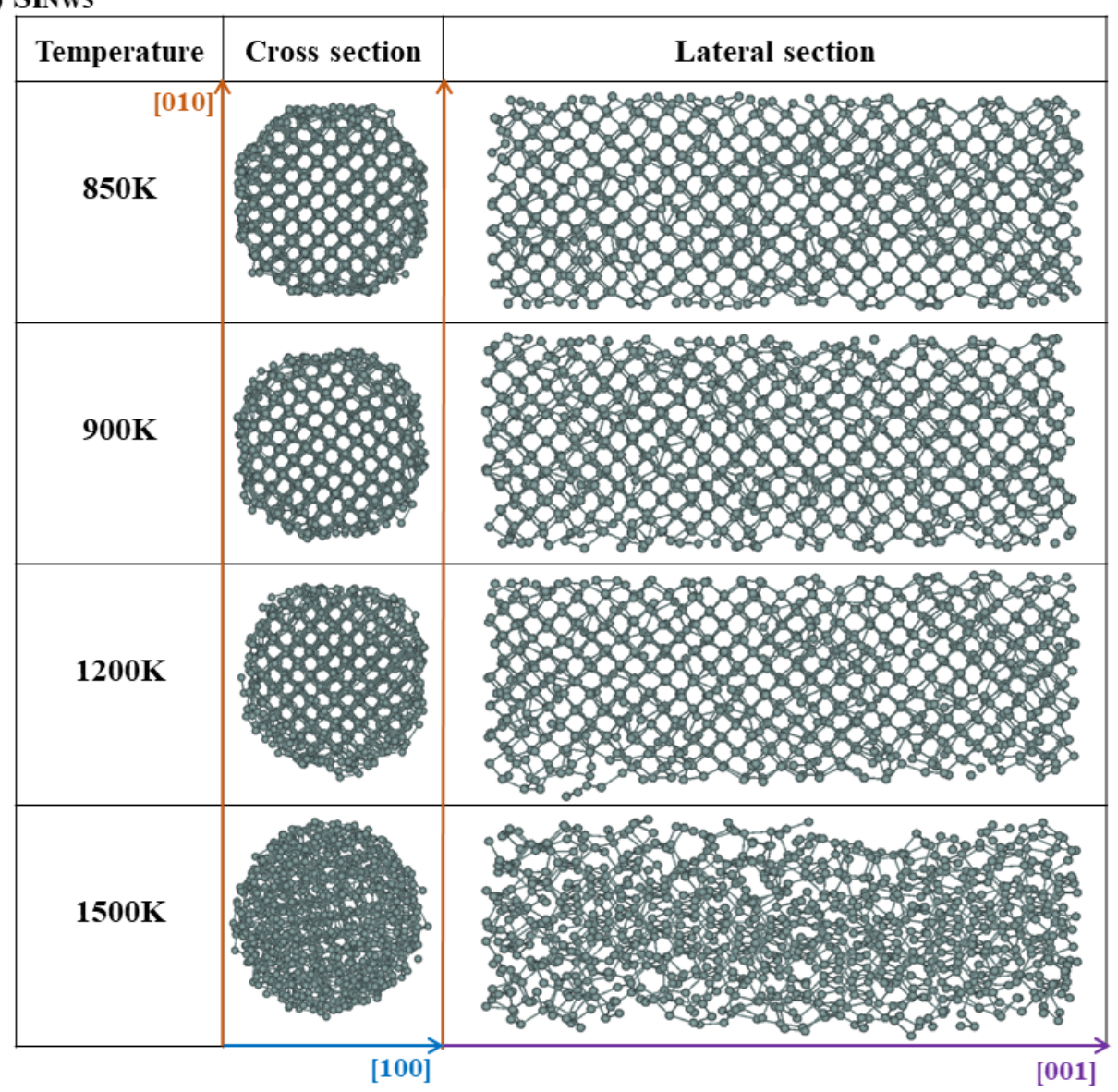

Figure 6. The atomic packing structures at different temperatures: (a) SiNW1, (b) SiNW2, (c) SiNW3.

Figure 7 shows the stress-strain curves of these nanowires subjected to uniaxial tensile loading at room temperature. As shown in this figure, nanowires start with a linear relation between stress and small strain, indicating that the nanowires undergo elastic deformation, where the stress-strain relation follows Hook's law. The slope of the stress-strain curve near zero strain provides Young's modulus. As the strain increases with a nonlinear trend, the stress begins to decrease, suggesting that the nanowires enter into plastic deformation. Until the strain reaches $0.11,0.11$ or 0.12 , the nanowire begins to yield, and the yield stress $\left(\sigma_{s}\right)$ is $1.5721,3.4126$ or $3.8959 \mathrm{GPa}$. Correspondingly, the packing images show that some of the surface atoms begin to separate, and some interior atoms leave their origin lattice positions. When the strain reaches $0.135,0.135$ or 0.15 , the stress of these nanowires reaches a maximum $\left(\sigma_{b}\right) 1.74296,3.77758$ or $4.11558 \mathrm{GPa}$; when the stress reaches the tensile strength, the plastic deformation stage is terminated. Then, there is an abrupt decrease in stress. The atomic packing images show that a necking phenomenon occurs in these wires. As shown in Figure 8, these three nanowires appear more light green to yellow for the atoms in the necking area than those outside of the area. These suggest that the pressure values in the necking area are mostly positive and the magnitude is higher than that of other regions of the atoms, which means most of these atoms are subjected to the compression due to the disordered atomic packing. For SiNW1, the atoms in the part outside of the necking area are blue to dark blue. For the atoms outside of the necking areas of SiNW2 and SiNW3, light green to light blue occurs, and even yellow is observed in the SiNW3. Therefore, these atoms in the remaining part of SiNW1 are in a stretched state, whereas a certain number of atoms in the remaining part of SiNW2 and SiNW3 are slightly compressed. 

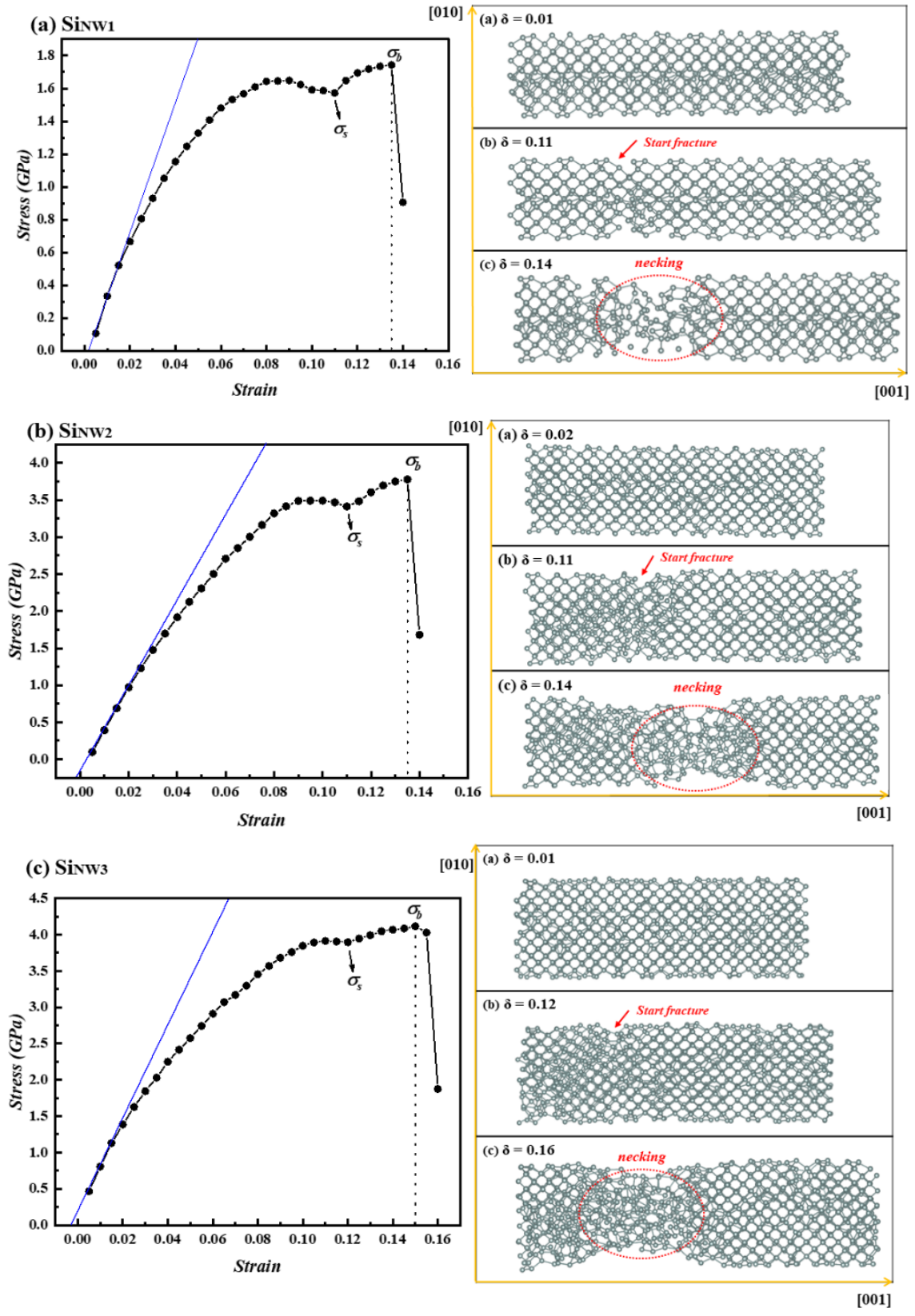

Figure 7. (Left) Stress-strain curves of silicon nanowires of various diameters at room temperatures; (Right) the structural evolution of nanowires: (a) SiNW1, (b) SiNW2, and (c) SiNW3. 


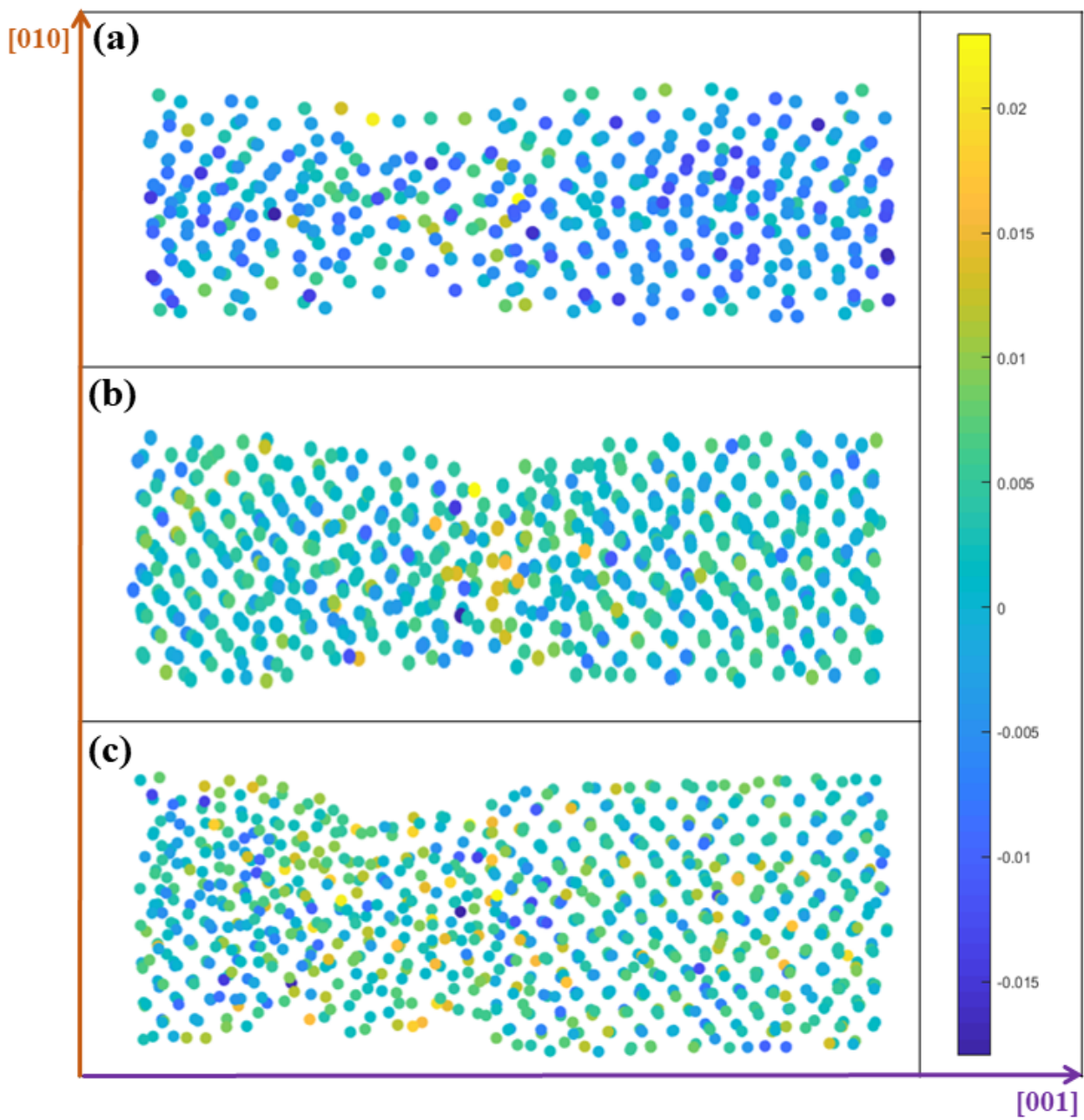

Figure 8. The isotropic atomic pressure in the three Si nanowires at $\sigma_{b}$ : (a) SiNW1, (b) SiNW2, (c) SiNW3.

As illustrated, the Young's modulus of the nanowires vary with the temperature in Figure 9; all of the nanowires tend to decrease with the increase in temperature. Due to the rearrangements of the surface atoms at different temperatures, the atomic motions and packing structures greatly affect the elasticity for these nanowires. Here, the modulus of the nanowire with a large cross-section area has a larger value than the small area. To observe the elasticity converge toward the bulk material, we further increased the diameters of the nanowires. The diameters were $1.629 \mathrm{~nm}, 2.429 \mathrm{~nm}, 3.259 \mathrm{~nm}$, and $3.541 \mathrm{~nm}$, which were labeled SiNW4, SiNW5, SiNW6 and SiNW7. Then, the nanowires were also subjected to a tensile test to calculate the Young's modulus. It can be seen from Figure 9 that when the diameter of the nanowire reaches $3.541 \mathrm{~nm}$, the Young's modulus still increases until it is close to the value of the bulk of Young's modulus, which is close to the value in about $109 \mathrm{GPa}$ [23]. 


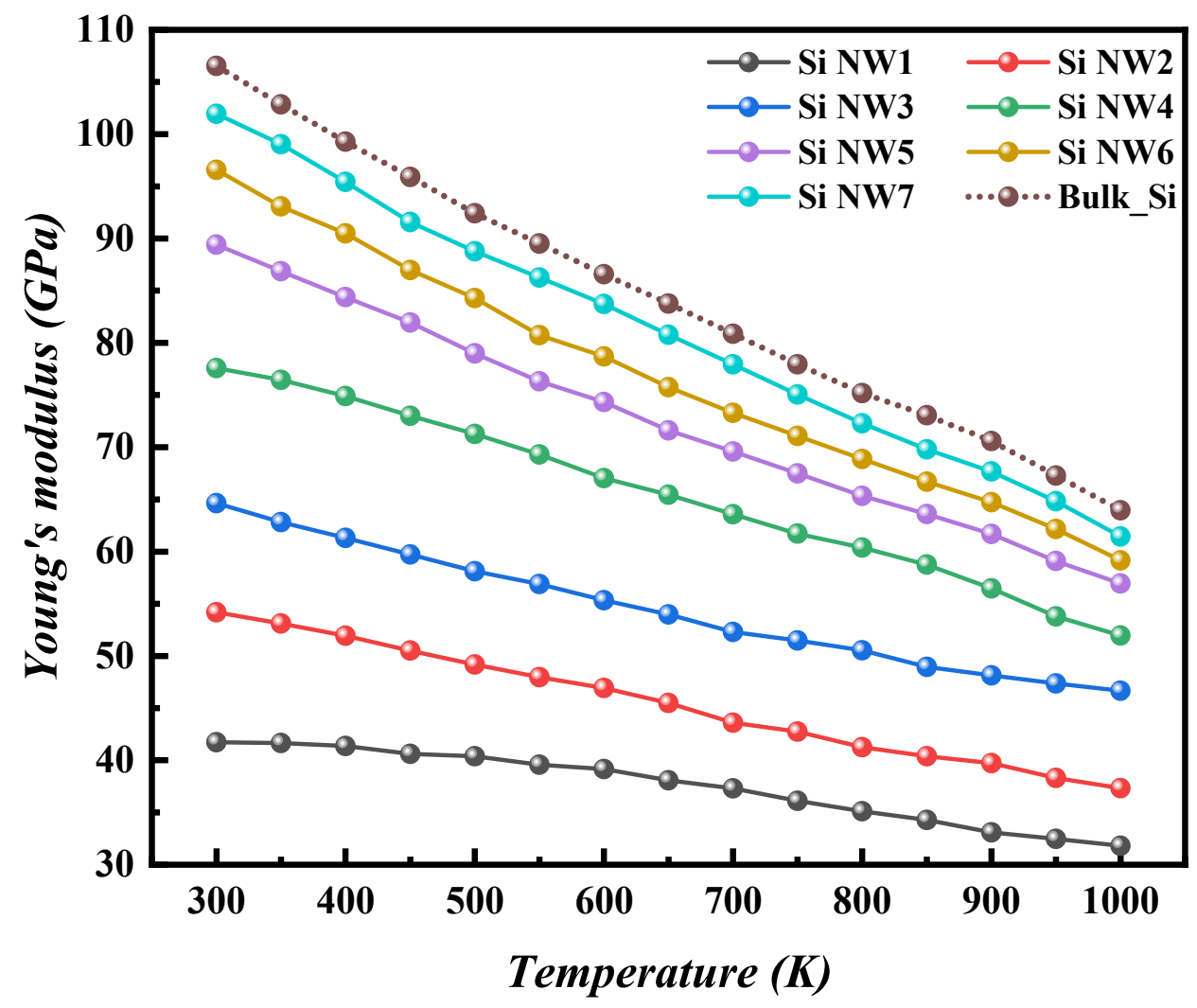

Figure 9. Young's modulus of Si NWs with different diameters and bulk Si varying from $300 \mathrm{~K}$ to $1000 \mathrm{~K}$.

\section{Conclusions}

In this paper, we investigated the structural and mechanical properties of the singlecrystalline silicon nanowires by using MD simulations with SW potential. The descriptions of our simulated systems performed by molecular dynamics were related to the temperature and tensile strength. These observations found that the packing changes come from the movements and rearrangements of surface atoms in a large temperature range. The wire diameter greatly affects the melting and tensile behaviors of these nanowires. For the wires with small cross-section area, continuous position adjustments ran through the heating range, whereas the wires with a large area presented a similar melting with to the bulk at a high temperature. The packing of surface atoms affected the melting and mechanical behaviors of those nanowires, especially for the wires with a small cross-section area. By performing uniaxial tensile simulations, the Si nanowires presented a mechanical behavior of significant plasticity including elastic deformation, yield, strain strengthening, and necking. For these nanowires, the atomic local stress in the necking area presented significant differences from those in the part outside of this area. The Young's modulus was temperature-dependent and size-dependent; as the temperature increased, the nanowires become soft.

Author Contributions: Conceptualization, D.Z. and L.Z.; methodology, D.Z.; software, D.Z.; validation, D.Z. and L.Z.; formal analysis, D.Z.; investigation, D.Z. and J.L.; data curation, D.Z. and J.L.; writingoriginal draft preparation, D.Z.; writing-review and editing, D.Z. and L.Z.; visualization, D.Z. and J.L.; supervision, L.Z. All authors have read and agreed to the published version of the manuscript.

Funding: This research received no external funding.

Data Availability Statement: Not applicable.

Acknowledgments: Authors wish to thanks the Project supported by the National Natural Science Foundation of China (Grant No. 51671051). 
Conflicts of Interest: The authors declare no conflict of interest.

\section{References}

1. Dasgupta, N.P.; Sun, J.; Liu, C.; Brittman, S.; Andrews, S.C.; Lim, J.; Gao, H.; Yan, R.; Yang, P. 25th Anniversary Article: Semiconductor Nanowires-Synthesis, Characterization, and Applications. Adv. Mater. 2014, 26, 2137-2184. [CrossRef]

2. Ahmad, R.; Mahmoudi, T.; Ahn, M.-S.; Hahn, Y.-B. Recent Advances in Nanowires-Based Field-Effect Transistors for Biological Sensor Applications. Biosens. Bioelectron. 2018, 100, 312-325. [CrossRef]

3. Buitrago, E.; Badia, M.F.-B.; Georgiev, Y.M.; Yu, R.; Lotty, O.; Holmes, J.D.; Nightingale, A.M.; Guerin, H.M.; Ionescu, A.M. Electrical Characterization of High Performance, Liquid Gated Vertically Stacked SiNW-Based 3D FET Biosensors. Sens. Actuators B Chem. 2014, 199, 291-300. [CrossRef]

4. $\quad$ Lee, S.; Kim, K.; Kang, D.-H.; Meyyappan, M.; Baek, C.-K. Vertical Silicon Nanowire Thermoelectric Modules with Enhanced Thermoelectric Properties. Nano Lett. 2019, 19, 747-755. [CrossRef] [PubMed]

5. Li, H.-P.; Zhang, R.-Q. Surface Effects on the Thermal Conductivity of Silicon Nanowires. Chin. Phys. B 2018, 27, 036801. [CrossRef]

6. Ziegler, A.; Luisier, M. Complex Band Structure Effects in k·p-BasedQuantum Transport Simulations of p-Type Silicon Nanowire Transistors. IEEE Trans. Electron Devices 2018, 65, 1298-1302. [CrossRef]

7. Čalnerytè, D.; Rimavičius, V.; Barauskas, R. Finite Element Analysis of Resonant Properties of Silicon Nanowires. Acta Mech. 2019, 230, 1907-1917. [CrossRef]

8. Zhou, G.; Xu, L.; Hu, G.; Mai, L.; Cui, Y. Nanowires for Electrochemical Energy Storage. Chem. Rev. 2019, 119, 11042-11109. [CrossRef] [PubMed]

9. Asthana, A.; Momeni, K.; Prasad, A.; Yap, Y.K.; Yassar, R.S. In Situ Observation of Size-Scale Effects on the Mechanical Properties of ZnO Nanowires. Nanotechnology 2011, 22, 265712. [CrossRef] [PubMed]

10. Liu, X.J.; Li, J.W.; Zhou, Z.F.; Yang, L.W.; Ma, Z.S.; Xie, G.F.; Pan, Y.; Sun, C.Q. Size-Induced Elastic Stiffening of ZnO Nanostructures: Skin-Depth Energy Pinning. Appl. Phys. Lett. 2009, 94, 131902. [CrossRef]

11. Wang, X.; Gu, Y.; Sun, X.; Wang, H.; Zhang, Y. Third-Order Elastic Constants of ZnO and Size Effect in ZnO Nanowires. J. Appl. Phys. 2014, 115, 213516. [CrossRef]

12. Cheng, G.; Miao, C.; Qin, Q.; Li, J.; Xu, F.; Haftbaradaran, H.; Dickey, E.C.; Gao, H.; Zhu, Y. Large Anelasticity and Associated Energy Dissipation in Single-Crystalline Nanowires. Nat. Nanotechnol. 2015, 10, 687-691. [CrossRef] [PubMed]

13. Yue, Y.; Liu, P.; Deng, Q.; Ma, E.; Zhang, Z.; Han, X. Quantitative Evidence of Crossover toward Partial Dislocation Mediated Plasticity in Copper Single Crystalline Nanowires. Nano Lett. 2012, 12, 4045-4049. [CrossRef] [PubMed]

14. Qin, Q.; Yin, S.; Cheng, G.; Li, X.; Chang, T.-H.; Richter, G.; Zhu, Y.; Gao, H. Recoverable Plasticity in Penta-Twinned Metallic Nanowires Governed by Dislocation Nucleation and Retraction. Nat. Commun. 2015, 6, 5983. [CrossRef] [PubMed]

15. Li, Z.; Wang, S.; Wang, Z.; Zu, X.; Gao, F.; Weber, W.J. Mechanical Behavior of Twinned SiC Nanowires under Combined Tension-Torsion and Compression-Torsion Strain. J. Appl. Phys. 2010, 108, 013504. [CrossRef]

16. Tang, D.-M.; Ren, C.-L.; Wang, M.-S.; Wei, X.; Kawamoto, N.; Liu, C.; Bando, Y.; Mitome, M.; Fukata, N.; Golberg, D. Mechanical Properties of Si Nanowires as Revealed by in Situ Transmission Electron Microscopy and Molecular Dynamics Simulations. Nano Lett. 2012, 12, 1898-1904. [CrossRef] [PubMed]

17. Han, X.D.; Zheng, K.; Zhang, Y.F.; Zhang, X.N.; Zhang, Z.; Wang, Z.L. Low-Temperature In Situ Large-Strain Plasticity of Silicon Nanowires. Adv. Mater. 2007, 19, 2112-2118. [CrossRef]

18. Gordon, M.J.; Baron, T.; Dhalluin, F.; Gentile, P.; Ferret, P. Size Effects in Mechanical Deformation and Fracture of Cantilevered Silicon Nanowires. Nano Lett. 2009, 9, 525-529. [CrossRef]

19. Kang, K.; Cai, W. Brittle and Ductile Fracture of Semiconductor Nanowires-Molecular Dynamics Simulations. Philos. Mag. 2007, 87, 2169-2189. [CrossRef]

20. Xu, W.; Kim, W.K. Molecular Dynamics Simulation of the Uniaxial Tensile Test of Silicon Nanowires Using the MEAM Potential. Mech. Mater. 2019, 137, 103140. [CrossRef]

21. Kang, K.; Cai, W. Size and Temperature Effects on the Fracture Mechanisms of Silicon Nanowires: Molecular Dynamics Simulations. Int. J. Plast. 2010, 26, 1387-1401. [CrossRef]

22. Gu, F.; Zhang, J.H.; Li, M.; Liu, L.Y.; Su, J. Influence of Defects on the Young's Modulus of [110] Silicon Nanowires with Different Cross Sections. Key Eng. Mater. 2015, 645-646, 151-156. [CrossRef]

23. Sun, H.; Chen, L.; Sun, S.; Zhang, T.-Y. Size- and Temperature-Dependent Young's Modulus and Size-Dependent Thermal Expansion Coefficient of Nanowires. Sci. China Technol. Sci. 2018, 61, 687-698. [CrossRef]

24. Wang, S.; Shan, Z.; Huang, H. The Mechanical Properties of Nanowires. Adv. Sci. 2017, 4, 1600332. [CrossRef] [PubMed]

25. Chang, T.-H.; Cheng, G.; Li, C.; Zhu, Y. On the Size-Dependent Elasticity of Penta-Twinned Silver Nanowires. Extrem. Mech. Lett. 2016, 8, 177-183. [CrossRef]

26. Haftel, M.I.; Gall, K. Density Functional Theory Investigation of Surface-Stress-Induced Phase Transformations in Fcc Metal Nanowires. Phys. Rev. B 2006, 74, 035420. [CrossRef]

27. Yao, Y.; Chen, S. Surface Effect in the Bending of Nanowires. Mech. Mater. 2016, 100, 12-21. [CrossRef]

28. Puglisi, R.A.; Garozzo, C.; Bongiorno, C.; Di Franco, S.; Italia, M.; Mannino, G.; Scalese, S.; La Magna, A. Molecular Doping Applied to Si Nanowires Array Based Solar Cells. Sol. Energy Mater. Sol. Cells 2015, 132, 118-122. [CrossRef] 
29. Abdul Rashid, J.I.; Abdullah, J.; Yusof, N.A.; Hajian, R. The Development of Silicon Nanowire as Sensing Material and Its Applications. J. Nanomater. 2013, 2013, 328093. [CrossRef]

30. Wang, D.; Sheriff, B.A.; McAlpine, M.; Heath, J.R. Development of Ultra-High Density Silicon Nanowire Arrays for Electronics Applications. Nano Res. 2008, 1, 9-21. [CrossRef]

31. Zhao, X.-J.; Seifert, G.; Zhu, J.; Zhang, D.-B. Twist-Induced Preferential Distribution of Dopants in Single-Crystalline Si Nanowires Phys. Rev. B 2019, 100, 174202. [CrossRef]

32. Wu, L.; Xu, X.; Zhang, L.; Qi, Y. Atomic Packing Characteristics and Electronic Structures of Si Nanowires from Density Functional Tight Binding Calculations. Superlattices Microstruct. 2019, 135, 106261. [CrossRef]

33. Stillinger, F.H.; Weber, T.A. Computer Simulation of Local Order in Condensed Phases of Silicon. Phys. Rev. B 1985, 31, 5262-5271. [CrossRef]

34. Schelling, P.K. Phase Behavior and Kinetics of a New Bond-Order Potential for Silicon. Comput. Mater. Sci. 2008, 44, 274-279. [CrossRef]

35. Gale, J.D.; Rohl, A.L. The General Utility Lattice Program (GULP). Mol. Simul. 2003, 29, 291-341. [CrossRef]

36. Sementa, L.; Barcaro, G.; Monti, S.; Carravetta, V. Molecular Dynamics Simulations of Melting and Sintering of Si Nanoparticles: A Comparison of Different Force Fields and Computational Models. Phys. Chem. Chem. Phys. 2018, 20, 1707-1715. [CrossRef] 